\title{
Mechanistic Evidence for Intermolecular Radical Carbonyl Additions Promoted by Samarium Diiodide
}

\author{
Anna Mette Hansen, ${ }^{\S}$ Karl B. Lindsay, ${ }^{\S}$ P. K. Sudhadevi Antharjanam, ${ }^{\ddagger}$ Jakob Karaffa, ${ }^{\S}$ Kim \\ Daasbjerg, ${ }^{\S}$ Robert A. Flowers ${ }^{\ddagger * \star}$ and Troels Skrydstrup ${ }^{\S *}$ \\ Contribution from the Center of Insoluble Protein Structures (inSPIN), Department of Chemistry, \\ Interdiscplinary Nanoscience Center, University of Aarhus, Langelandsgade 140, 8000 Aarhus C, Denmark, and \\ the Department of Chemistry, Lehigh University, Bethlehem, Pennsylvania 18015
}

\section{Supporting Information}

\section{Discussions related to reference 10}

The coupling yields with $N$-acyl oxazolidinones proved considerably less sensitive to the substitution pattern of both the alkyl acyl group and the electron deficient alkenes compared to similar coupling reactions performed with 4-pyridyl thioesters as the acyl radical equivalent. ${ }^{1}$ Second, an interesting regioselective divergence was observed in the reactions of the oxazolidinones $\mathbf{A}$ and $\mathbf{B}$ with $n$-butyl acrylate and $t$-butyl acrylamide not noted for any of the other substrates tested. Whereas reaction with the acrylamide provided the expected coupling products in

\section{Scheme i.}

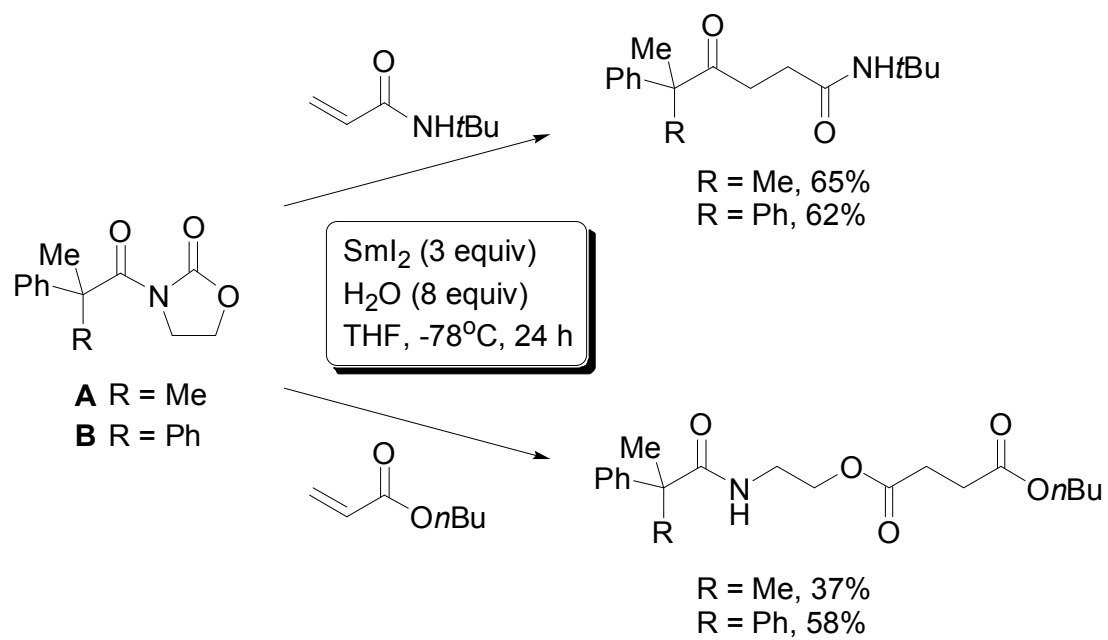

good yields, ${ }^{2} \mathrm{C}-\mathrm{C}$ bond formation with the acrylate took place with the oxazolidinone $\mathrm{C}=\mathrm{O}$ group (Scheme i). This latter result suggested that either electron transfer from $\mathrm{SmI}_{2}$ was rerouted to the carbonyl group of the heterocycle affording the ketyl radical anion $\mathbf{C}$ which was deemed highly improbable, or alternatively all these reactions were proceeding by initial reduction of the electron

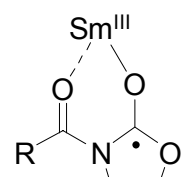

C

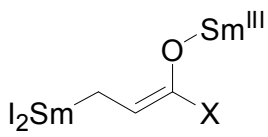

D

deficient alkenes to a dianion D followed by nucleophilic acyl substitution. Although not fully understood, the acrylate couplings would then appear to be more susceptible to steric factors. 
Nevertheless, the results described in this paper suggest that dianion $\mathbf{D}$ is not the reactive intermediate in the $\mathrm{C}-\mathrm{C}$ bond forming step.

The reduction potential for the cyclopropyl derivative 1 was measured to $E^{\text {red }}=-2.8 \mathrm{~V}$ vs SCE (1 $\mathrm{Vs}^{-1}$, THF). It is remarkable that any electron transfer from $\mathrm{SmI}_{2}\left(E^{\mathrm{o}}=-0.89 \mathrm{~V}\right.$ vs SCE, ref. 3) to this carbonyl group is observed considering the approx. $2 \mathrm{~V}$ difference. This clearly reflects the large inner-sphere nature of the electron transfer. Although water does increase the reducing abilities of $\mathrm{SmI}_{2}$ the effect is small. Water has only a small effect on the reducing abilities of $\mathrm{SmI}_{2}$ (ref. 4).

\section{Note Added in Proof}

One of the referees suggested that a competition experiment be performed with two different $\mathrm{N}$ acyloxazolidinones $(\mathrm{R}=t \mathrm{Bu}$ and $\mathrm{Me})$. An intramolecular process such as depicted in the intermediate $I$ (Scheme 5) should lead to a mixture of products, whereas an intermolecular reaction should involve only the less substituted reagent. A competition experiment was performed using the standard $\mathrm{SmI}_{2}$-coupling conditions described below with a 1:1:1 ratio of $N$-acetyl oxazolidinone, 7 and $t$-butyl acrylamide. The $t$-butyl ketone 9 was obtained in $78 \%$ yield with only traces of the corresponding methyl ketone. ${ }^{2}$ The $N$-acetyl oxazolidinone was almost completely recovered. This result is consistent with Scheme 5 proposing a cyclic intermediate. Since the $t$-butyl provides a more electron rich carbonyl (compared to the Me), chelation to 7 may activate it preferentially.

\section{General Methods}

The ${ }^{1} \mathrm{H}$ NMR spectra were recorded at $400 \mathrm{MHz}$ and ${ }^{13} \mathrm{C}$ NMR spectra were recorded at $100 \mathrm{MHz}$. The chemical shifts are reported in ppm downfield to TMS $(\delta=0)$ for ${ }^{1} \mathrm{H}$ NMR and relative to the central $\mathrm{CDCl}_{3}$ resonance $(\delta=77.16)$ for ${ }^{13} \mathrm{C}$ NMR. Solvents were dried according to standard procedures. Flash chromatography was carried out on Merck silica gel 60 (230-400 mesh). All reactions were carried out under an argon atmosphere.

\section{General procedure for the samarium diiodide couplings}

The $N$-acyl oxazolidinone was dissolved in THF, afterwhich water and either the acrylate or the acrylamide were added. The mixture was cooled to $-78^{\circ} \mathrm{C}$ and then $\mathrm{SmI}_{2}$ in THF $(0.1 \mathrm{M})$ was added dropwise over $10 \mathrm{~min}$. The mixture was stirred at $-78{ }^{\circ} \mathrm{C}$ for $24 \mathrm{~h}$, and then the flask was flushed with $\mathrm{O}_{2}$ to remove excess $\mathrm{SmI}_{2}$. Sat. aqueous $\mathrm{NH}_{4} \mathrm{Cl}(5 \mathrm{~mL})$ was added and the mixture was warmed to $20{ }^{\circ} \mathrm{C}$ and poured into $0.5 \mathrm{M} \mathrm{HCl}(40 \mathrm{~mL})$ before extraction with EtOAc $(3 \mathrm{x} 15 \mathrm{~mL})$. The combined organic portions were washed with sat. aqueous $\mathrm{Na}_{2} \mathrm{~S}_{2} \mathrm{O}_{3}(30 \mathrm{~mL})$, dried $\left(\mathrm{MgSO}_{4}\right)$, filtered and evaporated in vacuo. Pure product was obtained by flash column chromatography using the stated solvent system.

\section{Butyl 2-(2-methyl-2-phenylpropionylamino)ethyl succinate}

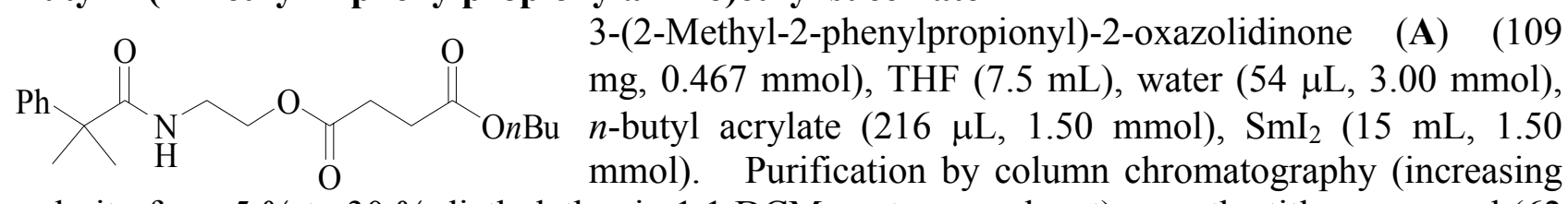
polarity from $5 \%$ to $30 \%$ diethylether in 1:1 DCM:pentane as eluant) gave the title compound (62 $\mathrm{mg}, 0.171 \mathrm{mmol}, 37 \%)$ as a colorless oil, and recovered 3-(2-methyl-2-phenylpropionyl)-2oxazolidinone (15 mg, $0.063 \mathrm{mmol}, 14 \%$ ) as a colorless solid.

${ }^{1} \mathrm{H}$ NMR $\left(400 \mathrm{MHz}, \mathrm{CDCl}_{3}\right) \delta(\mathrm{ppm}): 7.20-7.36(\mathrm{~m}, 5 \mathrm{H}), 5.60$ (br. t, $\left.J=5.2 \mathrm{~Hz}, 1 \mathrm{H}\right), 4.08(\mathrm{t}, J=$ $5.2 \mathrm{~Hz}, 2 \mathrm{H}), 4.03(\mathrm{t}, J=6.8 \mathrm{~Hz}, 2 \mathrm{H}), 3.40(\mathrm{q}, J=5.2 \mathrm{~Hz}, 2 \mathrm{H}), 2.49$ (AA'BB' system, 4H), 1.57 (quin, $J=7.2 \mathrm{~Hz}, 2 \mathrm{H}), 1.54(\mathrm{~s}, 6 \mathrm{H}), 1.34(\mathrm{hex}, J=7.6 \mathrm{~Hz}, 2 \mathrm{H}), 0.91(\mathrm{t}, J=7.6 \mathrm{~Hz}, 3 \mathrm{H}) .{ }^{13} \mathrm{C} \mathrm{NMR}$ 
$\left(100 \mathrm{MHz}, \mathrm{CDCl}_{3}\right) \delta(\mathrm{ppm}): 177.5,172.3,172.1,145.0,128.5$ (2C), $126.3(2 \mathrm{C}), 64.6,63.1,46.8$, 38.6, 30.5, 29.0, 28.9, 26.8 (2C), 19.0, 13.6. HRMS $\mathrm{C}_{20} \mathrm{H}_{29} \mathrm{NO}_{5}\left[\mathrm{M}+\mathrm{Na}^{+}\right]$; calculated 386.1944, found 386.1943 .

\section{Butyl 2-(2,2-diphenylpropionylamino)ethyl succinate}

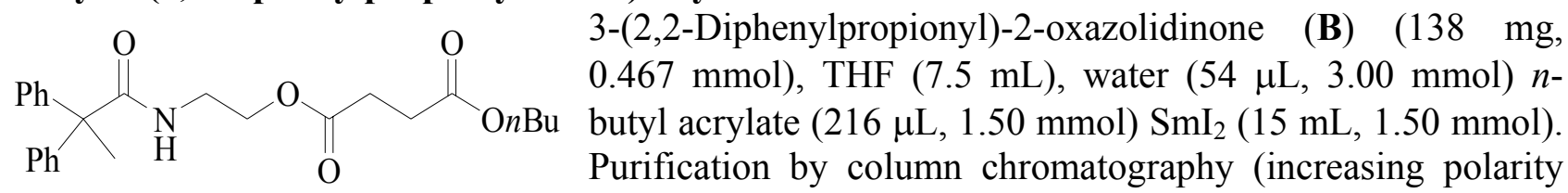
from $5 \%$ to $20 \%$ diethyl ether in $1: 1$ pentane:DCM as eluant) gave the title compound (115 mg, $0.270 \mathrm{mmol}, 58 \%$ ) as a colorless oil and recovered 3-(2,2-diphenylpropionyl)-2-oxazolidinone (57 $\mathrm{mg}, 0.193 \mathrm{mmol}, 41 \%)$ as a colorless solid.

${ }^{1} \mathrm{H}$ NMR (400 MHz, $\left.\mathrm{CDCl}_{3}\right) \delta(\mathrm{ppm}): 7.20-7.35$ (m, 10H), 5.82 (br. t, $\left.J=5.6 \mathrm{~Hz}, 1 \mathrm{H}\right), 4.14(\mathrm{t}, J=$ $5.6 \mathrm{~Hz}, 2 \mathrm{H}), 4.05(\mathrm{t}, J=6.8 \mathrm{~Hz}, 2 \mathrm{H}), 3.51(\mathrm{q}, J=5.6 \mathrm{~Hz}, 2 \mathrm{H}), 2.51$ (AA'BB' system, 4H), 1.99 (s, $3 \mathrm{H}), 1.59$ (quin, $J=7.2 \mathrm{~Hz}, 2 \mathrm{H}), 1.35$ (hex, $J=7.6 \mathrm{~Hz}, 2 \mathrm{H}), 0.93(\mathrm{t}, J=7.6 \mathrm{~Hz}, 3 \mathrm{H}) .{ }^{13} \mathrm{C} \mathrm{NMR}$ $\left(100 \mathrm{MHz}, \mathrm{CDCl}_{3}\right) \delta(\mathrm{ppm}): 175.2,172.1,172.0,144.8(2 \mathrm{C}), 128.4(4 \mathrm{C}), 128.0(4 \mathrm{C}), 126.9$ (2C), $64.6,63.1,56.9,38.8,30.5,29.0,28.9,26.9,19.0,13.6$. $\mathrm{HRMS}_{25} \mathrm{H}_{31} \mathrm{NO}_{5}\left[\mathrm{M}+\mathrm{Na}^{+}\right]$; calculated 448.2100 , found 448.2113 .

\section{n-Octyl 4-cyclopropyl-4-oxobutanamide (3) \\ $\mathrm{O}$}<smiles>O=CO</smiles>

$N$-Cyclopropanoyl-2-oxazolidinone (1) (25 mg, $0.16 \mathrm{mmol})$, $n$-octyl acrylate $(39 \mu \mathrm{L}, 0.19 \mathrm{mmol})$, THF $(3 \mathrm{~mL}), \mathrm{H}_{2} \mathrm{O}(23$ $\mu \mathrm{L}, 1.51 \mathrm{mmol}) \mathrm{SmI}_{2}(5.6 \mathrm{~mL}, 0.56 \mathrm{mmol})$. Purification by column chromatography (increasing polarity from 5 to $20 \%$

diethylether in 1:1 DCM:pentane as eluant) gave the title compound as a colorless oil (22 $\mathrm{mg}, 0.086$ mmol, $54 \%)$.

${ }^{1} \mathrm{H}$ NMR (400 MHz, $\left.\mathrm{CDCl}_{3}\right) \delta(\mathrm{ppm}): 4.05(\mathrm{t}, J=6.8 \mathrm{~Hz}, 2 \mathrm{H}), 2.79(\mathrm{t}, J=6.4 \mathrm{~Hz}, 2 \mathrm{H}), 2.58(\mathrm{t}, J=$ $6.4 \mathrm{~Hz}, 2 \mathrm{H}), 1.97-1.91(\mathrm{~m}, 2 \mathrm{H}), 1.64-1.57(\mathrm{~m}, 2 \mathrm{H}), 1.33-1.25(\mathrm{~m}, 10 \mathrm{H}), 1.05-1.01(\mathrm{~m}, 2 \mathrm{H}), 0.90-$ $0.85(\mathrm{~m}, 5 \mathrm{H}) .{ }^{13} \mathrm{C}$ NMR $\left(100 \mathrm{MHz}, \mathrm{CDCl}_{3}\right) \delta(\mathrm{ppm}): 208.8,173.0,65.0,37.8,31.9,29.4,29.3,28.8$, 28.3, 26.1, 22.8, 20.7, 14.2, 10.8 (2C). IR: 2927, 2855, 1734, $1700 \mathrm{~cm}^{-1}$. HRMS: $\mathrm{C}_{15} \mathrm{H}_{26} \mathrm{O}_{3}$ $\left[\mathrm{M}+\mathrm{Na}^{+}\right]$; calculated 277.1780 found 277.1783 .

\section{N-tert-Butyl 4-cyclopropyl-4-oxobutanamide (4)}

$\mathrm{O} \quad \mathrm{H} \quad \mathrm{N}$-Cyclopropanoyl-2-oxazolidinone (1) $(25 \mathrm{mg}, 0.19 \mathrm{mmol}), N$-tert-butyl acrylamide (35 mg, $0.27 \mathrm{mmol})$, THF ( $3 \mathrm{~mL}), \mathrm{H}_{2} \mathrm{O}(26 \mu \mathrm{L}, 1.44 \mathrm{mmol})$, $\mathrm{SmI}_{2}(6.3 \mathrm{~mL}, 0.63 \mathrm{mmol})$. Purification by column chromatography (increasing polarity from $25 \%$ to $33 \%$ EtOAc in pentane as eluant) gave

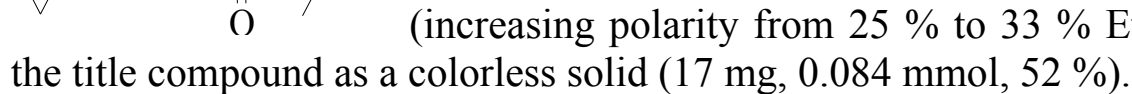

${ }^{1} \mathrm{H} \mathrm{NMR}\left(400 \mathrm{MHz}, \mathrm{CDCl}_{3}\right) \delta(\mathrm{ppm}): 5.50(\mathrm{br} \mathrm{s}, 1 \mathrm{H}), 2.90(\mathrm{t}, J=6.6 \mathrm{~Hz}, 2 \mathrm{H}), 2.35(\mathrm{t}, J=6.6 \mathrm{~Hz}$, $2 \mathrm{H}), 1.94(\mathrm{tt}, J=7.8,4.5 \mathrm{~Hz}, 1 \mathrm{H}), 1.66(\mathrm{~s}, 9 \mathrm{H}), 1.04-1.02(\mathrm{~m}, 2 \mathrm{H}), 0.90-0.86(\mathrm{~m}, 2 \mathrm{H}) .{ }^{13} \mathrm{C} \mathrm{NMR}$ $\left(100 \mathrm{MHz}, \mathrm{CDCl}_{3}\right) \delta(\mathrm{ppm}): 210.2,171.4,51.2,38.5,31.2,28.9$ (3C), 20.8, 10.9 (2C). IR: 3375, $3068,2965,1699,1668 \mathrm{~cm}^{-1}$. HRMS: $\mathrm{C}_{11} \mathrm{H}_{23} \mathrm{NO}_{2}\left[\mathrm{M}+\mathrm{Na}^{+}\right]$; calculated 220.1313 found 220.1310 .

\section{Di-n-octyl adipate (5)}

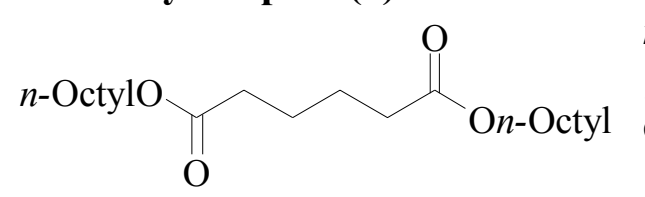

$n$-Octyl acrylate $(36 \mu \mathrm{L}, 0.174 \mathrm{mmol})$, THF $(3 \mathrm{~mL}), \mathrm{H}_{2} \mathrm{O}(25$ $\mu \mathrm{L}, 1.41 \mathrm{mmol}), \mathrm{SmI}_{2}(6.2 \mathrm{~mL}, 0.62 \mathrm{mmol})$. Purification by column chromatography (5\% EtOAc in pentane as eluant) 
gave the title compound as a colorless oil $(23 \mathrm{mg}, 0.061 \mathrm{mmol}, 70 \%)$.

${ }^{1} \mathrm{H}$ NMR $\left(400 \mathrm{MHz} \mathrm{CDCl}_{3}\right) \delta(\mathrm{ppm}): 4.05(\mathrm{t}, J=6.9 \mathrm{~Hz}, 4 \mathrm{H}), 2.36-2.28(\mathrm{~m}, 4 \mathrm{H}), 1.69-1.58(\mathrm{~m}$, $8 \mathrm{H}), 1.35-1.22(\mathrm{~m}, 20 \mathrm{H}), 0.88-0.95(\mathrm{~m}, 6 \mathrm{H}) .{ }^{13} \mathrm{C} \mathrm{NMR}\left(100 \mathrm{MHz}, \mathrm{CDCl}_{3}\right) \delta(\mathrm{ppm}): 173.6(2 \mathrm{C})$, 64.7 (2C), 34.1 (2C), 31.9 (2C), 29.4 (2C), 29.3 (2C), 28.8 (2C), 26.1 (2C), 24.6 (2C), 22.8 (2C), 14.2 (2C). IR: 2926, 2856, $1737 \mathrm{~cm}^{-1}$. HRMS: $\mathrm{C}_{22} \mathrm{H}_{42} \mathrm{O}_{4}\left[\mathrm{M}+\mathrm{Na}^{+}\right]$; calculated 393.2981, found 393.2991 .

n-Octyl 5,5-dimethyl-4-oxohexanoate (8)

$\mathrm{O}$<smiles>O=CO</smiles>

$N$-Pivaloyl-2-oxazolidinone (7) (30 $\mathrm{mg}, 0.18 \mathrm{mmol}), n$-octyl acrylate $(36 \mu \mathrm{L}, 0.173 \mathrm{mmol})$, THF $(3 \mathrm{~mL}), \mathrm{H}_{2} \mathrm{O}(26 \mu \mathrm{L}$, $1.39 \mathrm{mmol}), \mathrm{SmI}_{2}(6.2 \mathrm{~mL}, 0.62 \mathrm{mmol})$. Purification by column chromatography (20\% EtOAc in pentane as eluant)

gave the title compound as a colorless oil (36 mg, $0.133 \mathrm{mmol}, 74 \%)$.

${ }^{1} \mathrm{H} \mathrm{NMR}\left(400 \mathrm{MHz}, \mathrm{CDCl}_{3}\right) \delta(\mathrm{ppm}): 4.03(\mathrm{t}, J=6.8 \mathrm{~Hz}, 2 \mathrm{H}), 2.79(\mathrm{t}, J=6.5 \mathrm{~Hz}, 2 \mathrm{H}), 2.54(\mathrm{t}, J=$ $6.5 \mathrm{~Hz}, 2 \mathrm{H}$ ), 1.59 (quin, $J=6.8 \mathrm{~Hz}, 2 \mathrm{H}), 1.25-1.15(\mathrm{~m}, 10 \mathrm{H}), 1.14(\mathrm{~s}, 9 \mathrm{H}), 0.86(\mathrm{t}, J=6.8 \mathrm{~Hz}, 3 \mathrm{H})$. ${ }^{13} \mathrm{C}$ NMR $\left(100 \mathrm{MHz}, \mathrm{CDCl}_{3}\right) \delta(\mathrm{ppm}): 214.2,173.3,65.0,44.1,32.0,31.7,29.4,29.3,28.8,28.4$, 26.7 (3C), 26.1, 22.8, 14.3. IR: 2956, 2856, 2928, 1736, $1708 \mathrm{~cm}^{-1}$. HRMS: $\mathrm{C}_{16} \mathrm{H}_{30} \mathrm{O}_{3}\left[\mathrm{M}+\mathrm{Na}^{+}\right]$; calculated 293.2093 found 293.2104.

\section{(5S)- $\mathrm{N}$-tert-Butyl 5-benzyloxycarbonylamino-4-oxo-6-phenylhexanemide (13) ${ }^{2}$}

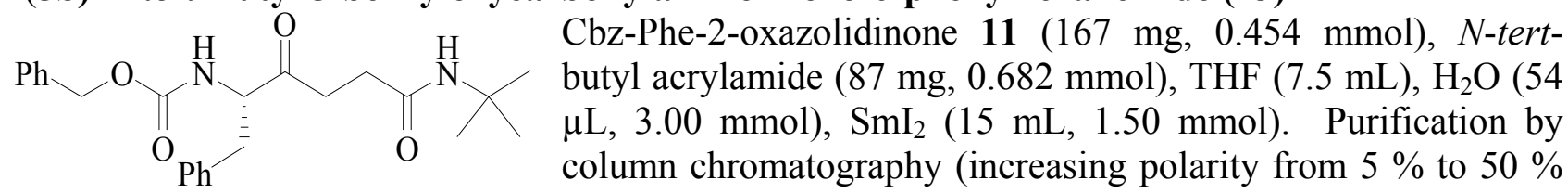
acetone in pentane as eluant) gave the title compound (109 $\mathrm{mg}, 0.265 \mathrm{mmol}, 58 \%)$ as a colorless solid. ${ }^{1}$

${ }^{1} \mathrm{H}$ NMR (400 MHz, $\left.\mathrm{CDCl}_{3}\right) \delta(\mathrm{ppm}) 7.36-7.22(\mathrm{~m}, 8 \mathrm{H}), 7.13(\mathrm{~d}, 2 \mathrm{H}, J=6.8 \mathrm{~Hz}), 5.38(\mathrm{~m}, 2 \mathrm{H})$, $5.08(\mathrm{~d}, 1 \mathrm{H}, J=12.4 \mathrm{~Hz}), 5.05(\mathrm{~d}, 1 \mathrm{H}, J=12.4 \mathrm{~Hz}), 4.60(\mathrm{q}, 1 \mathrm{H}, J=6.8 \mathrm{~Hz}), 3.18(\mathrm{dd}, 1 \mathrm{H}, J=$ 14.4, $6.0 \mathrm{~Hz}), 2.97(\mathrm{dd}, 1 \mathrm{H}, J=14.4,6.8 \mathrm{~Hz}), 2.88-2.73(\mathrm{~m}, 2 \mathrm{H}), 2.36(\mathrm{t}, 2 \mathrm{H}, J=6.4 \mathrm{~Hz}), 1.32(\mathrm{~s}$, 9H). ${ }^{13} \mathrm{C} \mathrm{NMR}\left(100 \mathrm{MHz}, \mathrm{CDCl}_{3}\right) \delta(\mathrm{ppm}) 208.1,170.7,155.9,136.4,136.1,129.4(2 \mathrm{C}), 128.7$ (2C), 128.6 (2C), 128.3, 128.1 (2C), 127.1, 67.0, 60.7, 51.4, 37.3, 35.5, 30.7, 28.9 (3C).

\section{Stopped-Flow Studies}

Kinetic experiments in THF were performed with a computer controlled SX-18 MV stopped-flow reaction spectrophotometer (Applied Photophysics Ltd. Surrey, UK). Solutions of $\mathrm{SmI}_{2} / \mathrm{H}_{2} \mathrm{O}$ and substrates ( $N$-pivaloyl oxazolidinone, $N$-tert-butyl acrylamide and $n$-butyl acrylate) were taken separately in airtight Hamilton syringes from a dry box and injected in to the stopped-flow system. The cell box and the drive syringes of the stopped flow reaction analyzer were flushed a minimum of three times with dry, degassed THF to make the system oxygen-free. The concentrations of $\mathrm{SmI}_{2}$ and $\mathrm{H}_{2} \mathrm{O}$ used for the study were $10 \mathrm{mM}$ and $40 \mathrm{mM}$, respectively. The concentration of the substrates was kept high relative to $\operatorname{SmI}_{2}(0.10 \mathrm{M})$ to maintain pseudo first-order conditions. Reaction rate constants were determined from exponential fitting the decays of $\mathrm{SmI}_{2}$ at $550 \mathrm{~nm}$. The decay of the $\mathrm{SmI}_{2}$ displayed first order behavior over $>4$ half-lives for $\mathrm{SmI}_{2}-N$-tert-butyl acrylamide and $n$-butyl acrylate combinations.

\section{References}

(1) (a) Blakskjær, P.; Høj, B.; Riber, D.; Skrydstrup, T. J. Am. Chem. Soc. 2003, 125, 4030. (b) Mikkelsen, L. M.; Jensen, C. M.; Høj, B.; Blakskjær, P.; Skrydstrup, T. Tetrahedron 2003, 59, 
10541. (c) Jensen, C. M.; Lindsay, K. B.; Andreasen, P.; Skrydstrup, T. J. Org. Chem. 2005, 70, 7512. (d) Lindsay, K. B.; Skrydstrup, T. Manuscript submitted for publication.

(2) Jensen, C. M.; Lindsay, K. B.; Taaning, R. H.; Karaffa, J.; Hansen, A. M. ; Skrydstrup, T. J. Am. Chem. Soc. 2005, 127, 6544.

(3) (a) Enemærke, R. J.; Daasbjerg, K.; Skrydstrup, T. Chem. Commun. 1999, 343. (b) Shabangi, M.; Kuhlman, M. L.; Flowers, R. A., II Org. Lett, 1999, 1, 2133. (c) Enemærke, R. J.; Hertz, T.; Skrydstrup, T.; Daasbjerg, K. Chem. Eur. J. 2000, 6, 3747.

(4) Prasad, E.; Flowers, R. A., II J. Am. Chem. Soc. 2005, 127, 18093. 